\title{
Conventional and retrospective change in health-related quality of life of trauma patients: an explorative observational follow-up study
}

\author{
Juanita A. Haagsma ${ }^{1 *}$, Inge Spronk ${ }^{1,2}$, Mariska A. C. de Jongh ${ }^{3}$, Gouke J. Bonsel ${ }^{1}$ and Suzanne Polinder ${ }^{1}$
}

\begin{abstract}
Background: Within trauma care measurement of changes in health-related quality of life (HRQL) is used in understanding patterns of recovery over time. However, conventionally-measured change in HRQL may not always reflect the change in HRQL as perceived by the patient. Recall bias and response shift may contribute to disagreement between conventional and retrospective change in HRQL. This study aimed to measure conventional and retrospective change of HRQL and assess to which extent recall bias and response shift contribute to disagreement between these two in a heterogeneous sample of adult trauma patients.
\end{abstract}

Methods: A sample of trauma patients ( $\geq 18$ years) who attended the Emergency Department and were admitted to an Intensive Care unit or ward of one of ten Dutch hospitals received postal questionnaires 1 week (T1) and 3 months (T2) post-injury. At T1 and T2 participants completed the EQ-5D-3 L and EQ-VAS for their current health status. At T2 participants also filled out a recall and then-test regarding their health status at $\mathrm{T} 1$. The responses were used to assess conventional and retrospective change, recall bias and response shift. Wilcoxon signed rank tests were used to examine conventional and retrospective change on a group level. The intraclass correlation coefficient (ICC) was used to examine individual agreement between conventional and retrospective change. Uni- and multivariate linear regression analysis were used to investigate the association between background factors and recall bias and response shift.

Results: The EQ-5D-3 L, recall and then-test were completed by 550 patients. Mean EQ-5D-3 L summary score improved from 0.48 at T1 to 0.74 at T2. Mean EQ-VAS score improved from 56 at T1 to 73 at T2. Retrospective change was significantly higher than conventional change (EQ-5D-3 L: $Z=-5.2, p<0.05$; EQ-VAS $Z=-2.1, p<0.05$ ). Pairwise comparisons showed that agreement between conventional and retrospective change was fair (EQ-5D-3 L: ICC $=0.49$; EQ-VAS: ICC = 0.48). For EQ-5-3 $\mathrm{L}$ response shift was significantly higher than recall bias $(Z=-4.5, p<0.05)$. Patients with traumatic brain injury (TBI), severe injury and/or posttraumatic stress symptoms were more susceptible to recall bias and response shift.

\footnotetext{
* Correspondence: j.haagsma@erasmusmc.nl

1 Department of Public Health, Erasmus MC University Medical Center Rotterdam, P.O. Box 2040, 3000, CA, Rotterdam, The Netherlands

Full list of author information is available at the end of the article
}

(c) The Author(s). 2020 Open Access This article is licensed under a Creative Commons Attribution 4.0 International License, which permits use, sharing, adaptation, distribution and reproduction in any medium or format, as long as you give appropriate credit to the original author(s) and the source, provide a link to the Creative Commons licence, and indicate if changes were made. The images or other third party material in this article are included in the article's Creative Commons licence, unless indicated otherwise in a credit line to the material. If material is not included in the article's Creative Commons licence and your intended use is not permitted by statutory regulation or exceeds the permitted use, you will need to obtain permission directly from the copyright holder. To view a copy of this licence, visit http://creativecommons.org/licenses/by/4.0/. The Creative Commons Public Domain Dedication waiver (http://creativecommons.org/publicdomain/zero/1.0/) applies to the data made available in this article, unless otherwise stated in a credit line to the data. 
(Continued from previous page)

Conclusions: We conclude that, compared to recall bias, response shift contributed more to the disagreement between conventional and retrospective change in EQ-5D-3 L summary score and EQ-VAS. Predictable subgroups of trauma patients were more susceptible to recall bias and response shift.

Keywords: Health-related quality of life, Mental recall, Wounds and injuries, Brain injuries traumatic

\section{Background}

A well-studied outcome in trauma care is health-related quality of life (HRQL) [1]. HRQL is used to estimate the impact of an injury on a patient's life, and enables to evaluate quality of care in patients [2]. Measurement of change in HRQL, individual or aggregate, has been used to evaluate health interventions in a wide range of conditions and populations (e.g. [3-7]). Inaccurate measurement of change in HRQL may therefore affect clinical practice and health care, and ultimately the quality of care and HRQOL of patients. In an observational context this change provides insight into patterns of recovery over time $[8,9]$. The understanding of recovery patterns supports the clinician in setting expectations, and the timely identification of specific patient groups with lower HRQL over time. Knowing who faces a poor prognosis may guide the development and application of targeted interventions to halt this development.

However, conventionally-measured change in HRQL may not always reflect the change in HRQL as perceived or experienced by the patient. Conventionally-measured change in HRQL is defined by the difference between the direct measurements of HRQL at two consecutive occasions. The patient's perceived change in HRQL is defined as the difference between the directly measured current HRQL and the HRQL as stated by the patient to be the HRQL on a specified previous occasion. Indeed, McPhail showed that agreement between conventional change and retrospective change in HRQL was not strong. A large proportion of the disagreement was attributed to so-called recall bias [10]. Recall bias is defined as a systematic measurement error, due to memory decay, that is the fading of memory with time. From the current standpoint, past health may be memorized as more deteriorated or better than it actually was; the direction depending on psychological mechanisms which keep better or worse memories better alive [11]. The magnitude of recall bias may depend on the scale that is used to measure HRQL, where subjective scales, such as the visual analogue scale (VAS), may easier be distorted than classification-like scales, like the EQ-5D $[12,13]$. Recall bias of HRQL has been observed among patients with e.g. multiple sclerosis, psoriasis, cancer, injury and total hip arthroplasty [14-17] A study among patients with traumatic brain showed that recall bias was stronger in patients with high symptoms, which include memory problems [18]. This indicates that the size of recall bias may be higher among patients who experience memory problems compared to their counterparts.

Response shift may also contribute to disagreement between conventional and retrospective change in HRQL. Response shift is a true change of a patients' perspective towards the targeted construct, caused by a change in internal standards, a change in values, and/or a redefinition of the construct $[19,20]$. This may change the direction of change. Among trauma patients response shift may occur between multiple post-injury HRQL measurements due to patients adapting to their ill health $[19,20]$. However, the magnitude of response shift may vary across type and severity of injury. A study among multiple sclerosis patients showed that being more disabled was associated with a change in internal standards with regards to certain HRQL dimensions [21], indicating that response shift may be stronger among patients with more severe injuries. Consequently, the contribution of response shift to disagreement between conventional and retrospective change may also vary across subgroups of trauma patients.

McPhail et al. were the first to investigate response shift and recall bias simultaneously in a sample of 101 elderly hospitalized patients [10]. The investigators argued that the contribution of response shift and recall bias may vary across other patient groups. This may particularly be the case for trauma patients, since injuries comprise of heterogeneous patterns of ill-health and may affect patients of all age groups.

The aims of this study were to measure conventional and retrospective change of HRQL, measured with the EQ-5D-3 L and the EQ-VAS, and to assess to which extent recall bias and response shift contribute to disagreement, in a heterogeneous sample of trauma patients.

\section{Hypotheses}

We tested the following hypotheses:

- Agreement between conventional and retrospective change of HRQL, as measured with EQ-VAS, is lower compared to the agreement if HRQL was measured with EQ-5D-3 L, because recall bias and response shift more easily distort subjective scales (like a VAS) than a classification-like scale like EQ5D-3 L. 
- Agreement between conventional and retrospective change of HRQL is higher among trauma patients with less severe injuries (ISS $<16$ ), because low impact trauma requires less adaptation to ones (final) health status compared severe trauma.

- Recall bias rather than response shift causes disagreement between conventional and retrospective change because with the time lapse chosen ( 3 months) memory problems affecting recall are no longer trivial.

- In older patients, in patients with traumatic brain injury (TBI) and patients with posttraumatic stress disorder (PTSD) the size of recall bias is higher compared to their counterparts, because these patients experience more memory problems.

\section{Methods}

\section{Study design}

This study utilizes data from a registry-based study on injury patients in Noord-Brabant (2.5 M inhabitants), the Netherlands. This prospective longitudinal cohort study, called the Brabant Injury Outcome Surveillance (BIOS) study, assessed outcomes in trauma patients, admitted to one of the ten hospitals in the Noord-Brabant region in the Netherlands [22]. The BIOS study includes multiple HRQL measurements up to 24 months after injury. Response shift items and recall questions were intentionally included in the 3 month follow-up survey. Ethical approval for the observational data analysis of this study was received from the Medical Ethics Committee Brabant (NL50258.028.14).

\section{BIOS study population}

All trauma patients ( $\geq 18$ years), who attended the Emergency Department (ED) and were admitted to an Intensive Care unit (ICU) or ward of one of the ten hospitals between November 2015 and November 2016, and who were discharged alive, qualified for inclusion. Patients were excluded if they were unable to understand or answer Dutch language questionnaires, when they had a pathological fracture due to a primary malignancy, or when they had no permanent address [22].

The eligible patients were invited to participate in the BIOS study via a postal invitation one week after admission to hospital. This invitation was accompanied by an informed consent form and the first survey (T1). For this procedure ethical permission is obtained. Nonresponders received a telephone call to discuss their participation. The 3 month follow up survey (T2) was sent to the patient if consent and the completed T1 survey were received by the researchers. For present study, we included data from patients who completed both surveys.

\section{Self-report measures}

T1 included questions on patient characteristics (e.g. age and gender), and 19 items regarding the presence of one or more chronic diseases (e.g. diabetes) prior to the injury to assess comorbidity [23]. If a patient suffered from one or more chronic disease(s) additional to the injury that qualified for inclusion, he/she was defined as having comorbidity [24]. Level of education was divided in three categories: low, middle or high. For patients classified as low level the highest level of education obtained was no education, primary school or prevocational education. Patients classified as middle level followed at best secondary or vocational education, and patients classified as high completed professional higher education or university level. Both surveys (T1, T2) included the EQ-5D-3 L.

The EQ-5D-3 L is a standardized generic HRQL measure [25]. The EQ-5D-3 L covers five dimensions: mobility, self-care, usual activities, pain/discomfort, and anxiety/depression and a visual analogue scale (EQVAS) [25]. The five dimensions have three response options: no problems, moderate problems, extreme problems [26]. The ordinal scores on the dimensions can be used in a descriptive analysis, but may also be used as an input to calculate an EQ-5D-3 L summary score combining all dimensions, ranging from 0 (death) and 1 (full health) [27]. For few health states considered worse than death the summary score can have a value lower than zero. The EQ-VAS consists of a scale from 0 (worst imaginable health) to 100 (best imaginable health) and measures the patient's self-rated health in a subjective way. Apart from the complete EQ-5D-3 L, the T2 questionnaire also included the so-called 'recall' and the 'then' test. The recall test asked patients to report what they remember to have reported on the EQ-5D-3 L on the previous occasion (T1). The then test asked patients to report how they believe now what their health status was at previous assessment (T1). Both the recall test and then test consisted of six items: five EQ-5D-3 L items and the EQ-VAS.

The T2 survey also included the impact of event scale (IES) [28]. The IES is a validated self-report instrument that uses 15 -items questionnaire to assess stress symptoms caused by a traumatic event. Each item is scored on a 4-point scale $(0,1,3,5$ points), where 0 refers to "not at all" and 5 refers to "extremely". The total IESscore ranges from 0 (no meaningful impact into any direction) to 75 (severe impact event on all 15 items). PTSD is assumed to be present if IES-score exceeds 35 [29].

\section{Injury data}

Apart from the self-report data, clinical injury data of included trauma patients were available from the Brabant Trauma Registry. All BIOS hospitals also participate in 
this registry. Injury data comprised the Injury Severity Score (ISS) [30] and the Abbreviated Injury Scale (AIS) [31]. The AIS classifies the severity of a trauma via an anatomic scale and it scores the type, location and severity of each injury that was sustained by a patient. The AIS score of the three most severely injured body regions are squared and summed to an ISS. The ISS is an accepted summary score for the severity of a trauma, and ranges from 1 to 75 . A major trauma is assumed to be present if the ISS exceeds 15 [32]. The ISS was automatically calculated based on the AIS scores that were registered in the Brabant Trauma Registry.

\section{Data analysis}

SPSS version 23 was used for all analyses. We performed a non-response analysis to study whether responders differed from non-responders. Mann Whitney $U$ tests were used for continuous variables and Chi-square tests for categorical variables. Descriptive statistics were used to assess the sample characteristics, and EQ-5D-3 L dimension, EQ-5D-3 L summary scores and EQ-VAS scores.

T1 health outcomes were compared between subgroups: males vs. females, age $<65$ vs. $\geq 65$ years, absence vs. presence of pre-existing comorbidity, absence vs. presence of traumatic brain injury (TBI), ISS $<16$ vs. ISS $\geq 16$, and absence vs. presence of PTSD using Mann Whitney U tests. Similarly, Kruskal Wallis test was used to compare outcomes according to educational attainment (three levels).

The following equations were used to calculate conventional and retrospective change in HRQL:

$$
\begin{aligned}
& \text { Conventional change }_{\mathrm{EQ}-5 \mathrm{D} \text { summary score } \mathrm{T} 1, \mathrm{~T} 2}=\mathrm{EQ}-5 \mathrm{D}+\mathrm{BL} \\
& \text { summary score }_{\mathrm{T} 2}-\mathrm{EQ}-5 \mathrm{D}-3 \mathrm{~L} \text { summary score } \\
& \mathrm{T} 1
\end{aligned}
$$

Where EQ-5D-3 L summary score ${ }_{\mathrm{T} 2}$ and EQ-5D-3 L summary score T1 $_{1}$ are the directly measured EQ-5D-3 L at $\mathrm{T} 1$ and $\mathrm{T} 2$, respectively.

$$
\text { Conventional change } \mathrm{EQ}_{\mathrm{EAS} \mathrm{T} 1 \mathrm{~T} 2}=\mathrm{EQ}-\mathrm{VAS}_{\mathrm{T} 2}-\mathrm{EQ}-\mathrm{VAS}_{\mathrm{T} 1}
$$

Where EQ-VAS $\mathrm{T}_{2}$ and EQ-VAS 1 are the directly measured EQ-VAS scores at T1 and T2, respectively.

$$
\begin{aligned}
& \text { Retrospective change }_{\mathrm{EQ}-5 \mathrm{D} \text { summary score } \mathrm{T} 1, \mathrm{~T} 2}=\mathrm{EQ}-5 \mathrm{D}-3 \mathrm{~L} \\
& \text { summary score }_{\mathrm{T} 2}-\mathrm{EQ}-5 \mathrm{D}-3 \mathrm{~L} \text { then test }
\end{aligned}
$$

Where EQ-5D-3 L summary score T2 $_{2}$ is the directly measured EQ-5D-3 L at T2 and EQ-5D-3 L then test is the EQ-5D-3 L summary score of how the respondents believed their EQ-5D-3 L health status was at previous assessment (T1).

$$
\text { Retrospective change }_{\mathrm{EQ}-\mathrm{VAS} \mathrm{T} 1, \mathrm{~T} 2}=\mathrm{EQ}-\mathrm{VAS}_{\mathrm{T} 2}-\mathrm{EQ}-\mathrm{VAS} \text { then test }
$$

Where EQ-VAS $\mathrm{T}_{2}$ is the directly measured EQ-VAS score at T2 and EQ-VAS then test is the EQ-VAS score of how the respondents believed their EQ-VAS score was at previous assessment (T1).

See Fig. 1 for a schematic overview of the calculations of conventional and retrospective change, recall bias and response shift.

Wilcoxon signed rank tests were used to compare conventional and retrospective change in EQ-5D-3 L summary scores and EQ-VAS scores for the total sample as well as for subgroups (males vs. females, age $<65$ vs. $\geq 65$ years, absence vs. presence of pre-existing comorbidity, absence vs. presence of traumatic brain injury (TBI), ISS $<16$ vs. ISS $\geq 16$, and absence vs. presence of PTSD). It was examined whether conventional and retrospective change in EQ-5D-3 L summary scores and EQ-VAS scores differed for the different subgroups, and whether this was in accordance to our hypotheses. We calculated the intraclass correlation coefficient (ICC) to assess agreement between conventional and retrospective change on patient level [33]. We calculated the ICC for the whole group, and for subgroups (age, gender, educational level, comorbidity status, ISS category and PTSD symptoms). ICC was defined as poor $(<0.40)$, fair $(0.40-0.59)$, good $(0.60-0.74)$ or excellent $(0.75-1.00)$ [34].

Recall bias and response shift were calculated with the following equations:

$$
\begin{aligned}
& \text { Recall bias }_{\mathrm{EQ}-5 \mathrm{D} \text { summary score }}=\mathrm{EQ}-5 \mathrm{D}-3 \mathrm{~L} \text { recall test }- \\
& \text { EQ-5D-3L summary score } \\
& \mathrm{T} 1
\end{aligned}
$$

Where EQ-5D-3 L recall test is the EQ-5D-3 L summary score of the EQ-5D-3 L health status that the respondents remember to have reported at the previous assessment (T1) and EQ-5D-3 $\mathrm{L}$ summary score $\mathrm{T}_{1}$ is the directly measured EQ-5D-3 L at T1.

$$
\text { Recall bias }_{\mathrm{EQ}-\mathrm{VAS}}=\mathrm{EQ}-\mathrm{VAS} \text { recall test }-\mathrm{EQ}-\mathrm{VAS}_{\mathrm{T} 1}
$$

Where EQ-VAS recall test is the EQ-VAS score that the respondents remember to have reported at the previous assessment (T1) and EQ-VAS $\mathrm{T}_{1}$ is the directly measured EQ-VAS at T1. 


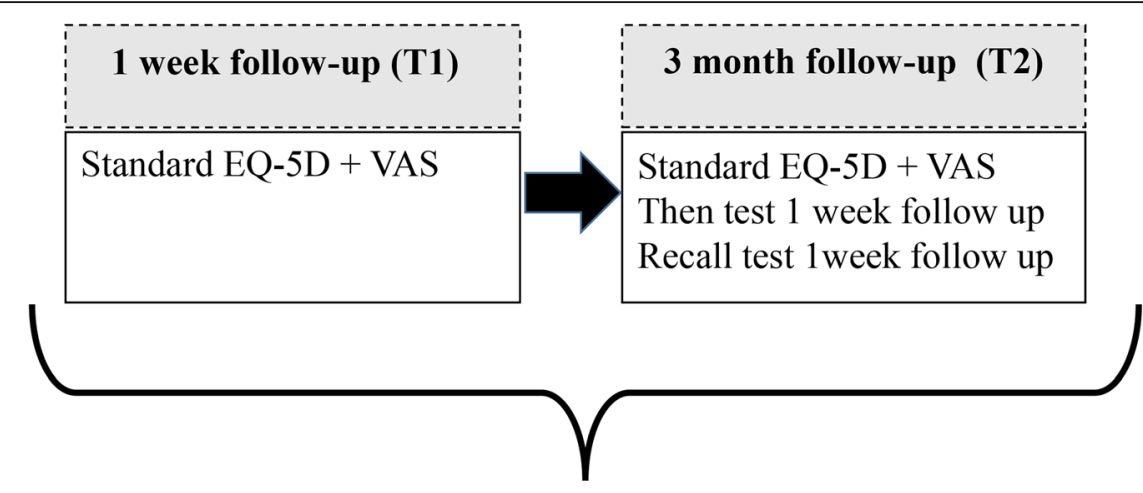

$$
\begin{aligned}
& \text { - Conventional change }=\text { standard EQ-5D at T2 }- \text { standard EQ-5D at T1 } \\
& \text { - Retrospective change }=\text { standard EQ-5D at T2 }- \text { then test } \\
& \text { - Recall bias }=\text { recall test }- \text { standard EQ-5D at T1 } \\
& \text { - Response shift }=\text { then test }- \text { standard EQ-5D at T1 }
\end{aligned}
$$

Fig. 1 Schematic overview of the calculations of conventional and retrospective change, recall bias and response shift

Response shift $\mathrm{EQ}_{\mathrm{E}-5 \mathrm{D} \text { summary score }}=\mathrm{EQ}-5 \mathrm{D}-3 \mathrm{~L}$ then test -

EQ-5D-3 L summary score T1 $_{1}$

Where EQ-5D-3 L then test is the EQ-5D-3 L summary score of how the respondents believed their EQ$5 \mathrm{D}-3 \mathrm{~L}$ health status was at previous assessment (T1) and EQ-5D-3 $\mathrm{L}$ summary score ${ }_{\mathrm{T} 1}$ is the directly measured EQ-5D-3 L at T1.

$$
\text { Response shift }_{\mathrm{EQ}-\mathrm{VAS}}=\mathrm{EQ}-\mathrm{VAS} \text { then test }-\mathrm{EQ}-\mathrm{VAS}_{\mathrm{T} 1}
$$

Where EQ-VAS then test is the EQ-VAS score of how the respondents believed their health status was at previous assessment (T1) and EQ-VAS $\mathrm{T}_{1}$ is the directly measured EQ-VAS at T1.

Wilcoxon signed rank tests were used to examine the differences between response shift and recall bias. Differenced were also studied on a subgroup level in order to examine whether recall bias and response shift differs between the subgroups defined. To estimate the role of background factors in recall bias and response shift respectively, we predicted recall bias and response shift from the socio-demographic factors (age, gender, education), TBI (yes or no), injury severity level (ISS as a continuous variable) and PTSD symptoms (IES-score as a continuous variable). Straightforward univariate and multivariate linear regression analysis were applied, with backward selection (deselection criterion $p<0.10$ ) were used to investigate the association between sociodemographics, comorbidity, TBI, injury severity, PTSD and recall bias and response shift.
Overall $p$-values $<0.05$ were considered to indicate statistical significance, although our analysis primarily was explorative.

\section{Results}

\section{Study population}

In total, 1518 of the 5731 invited patients participated in the BIOS study (26.5\%). Responders were significantly younger than non-responders $(p<0.05)$ and significantly more often male than non-respondents $(p<0.05)$. In total, 790 patients responded on the T1 survey and 1351 patients responded on the T2 survey. However, only 550 of these patients completed the EQ-5D-3 L and EQ-VAS at $\mathrm{T} 1$ and $\mathrm{T} 2$, the then-test EQ-5D-3 L and EQ-VAS (at T2) and the recall EQ-5D-3 L and EQ-VAS (at T2) and were therefore included in this study. These $550 \mathrm{com}$ pleters were significantly more often male, significantly younger, higher educated and had a shorter hospital stay compared to non-completer. The completers had a mean age of 61.0 years (SD 16.0) and slightly more than half of the participants (56.0\%) was male (Table 1). Most participants had a middle or high-level education and comorbidity was highly prevalent $(56.2 \%)$. Patients' median hospital stay was 4.0 (IQR 2.0-6.0) days, and most common injuries were mild traumatic brain injury (28.9\%) and hip fracture (20.7\%). Median ISS was 5.0 (IQR 4.0-9.0).

\section{EQ-5D-3 L - conventional change versus retrospective change}

Table 2 shows the mean EQ-5D-3 L summary score at $\mathrm{T} 1$ and T2, mean Then Test, mean conventional change and retrospective change between EQ-5D-3 L summary 
Table 1 Characteristics of study population

\begin{tabular}{ll}
\hline Characteristic & $\begin{array}{l}\text { Study population } \\
(\boldsymbol{n}=\mathbf{5 5 0})\end{array}$ \\
\hline Gender: Male & $308(56.0 \%)$ \\
Age $($ Mean, SD) & $61.0(16.0)$ \\
Education & \\
Low & $127(23.1 \%)$ \\
Middle & $214(38.9 \%)$ \\
High & $199(36.2 \%)$ \\
Unknown & $10(1.8 \%)$
\end{tabular}

\section{Comorbidity status}

No comorbidity
Comorbidity
Unknown

231 (42.0\%)

$309(56.2 \%)$

$10(1.8 \%)$

$4.0(2.0-6.0)$

\section{Length of hospital stay in days (Median, IQR)}

Number of injuries

1
2
$\geq 3$

$275(50.0 \%)$
$129(23.5 \%)$
$146(26.5 \%)$

Type of injury ${ }^{b}$

Mild TBI
Hip fracture
Pelvic injury
Rib fracture
Tibia, complex foot or femur fracture
Shoulder and upper arm injury
Stable vertebral fracture or disc injury
Radius, ulna or hand fracture
Thoracic injury
Facial fracture
Severe TBI
Mild abdominal injury
Severe abdominal injury
Spinal cord injury

Injury Severity Score

$<8$

$320(58.2 \%)$

8-16

$\geq 16$

Unknown

\section{PTSD symptoms ${ }^{a}$}

No PTSD (IES < 35)
PTSD (IES $\geq 35)$
Unknown

$451(82.0 \%)$

$41(7.5 \%)$

$58(10.5 \%)$

\section{EQ-5D-3 L scores at T1}

Utility score (Mean, SD)

EQ-VAS (Mean, SD)
Table 1 Characteristics of study population (Continued)

\begin{tabular}{ll}
\hline Characteristic & $\begin{array}{l}\text { Study population } \\
(\boldsymbol{n}=\mathbf{5 5 0})\end{array}$ \\
\hline $\begin{array}{l}\text { Mobility (\% reporting moderate or } \\
\text { extreme problems) }\end{array}$ & $72.0 \%$ \\
$\begin{array}{l}\text { Self-care (\% reporting moderate or } \\
\text { extreme problems) }\end{array}$ & $69.8 \%$ \\
$\begin{array}{l}\text { Usual activities (\% reporting moderate or } \\
\text { extreme problems) }\end{array}$ & $85.5 \%$ \\
$\begin{array}{l}\text { Pain/discomfort (\% reporting moderate or } \\
\text { extreme problems) }\end{array}$ & $88.0 \%$ \\
$\begin{array}{l}\text { Anxiety/depression (\% reporting moderate } \\
\text { or extreme problems) }\end{array}$ & $24.9 \%$ \\
\hline
\end{tabular}

Note. $S D$ standard deviation, IQR inter quartile range

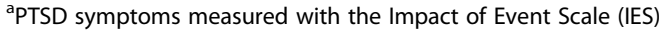

3 months post-injury

${ }^{\mathrm{b}}$ The total number of patients by type of injury exceeds 550 , because many respondents had multiple injuries

scores at T1 and T2. Mean EQ-5D-3 L summary scores at $\mathrm{T} 1$ and $\mathrm{T} 2$ were 0.482 (SD 0.30) and 0.735 (SD 0.24), respectively. A lower EQ-5D-3 L summary score at $\mathrm{T} 1$ was associated with being younger, having an ISS $\geq 16$, not having a TBI and having PTSD three months postinjury (all $p<0.05$ ). Pairwise comparisons showed that agreement between retrospective and conventional change was fair (ICC $=0.49, p<0.05$ ) (see Table 2). Retrospective change was significantly higher compared to conventional change $(\mathrm{Z}=-5.2, p<0.05)$. The difference between conventional and retrospective change was highest among patients with ISS $\geq 16$ (mean difference $=$ $-0.12, \mathrm{Z}=-1.9, p=0.058)$.

\section{EQ-5D-3 $L$ - recall bias versus response shift}

Recall bias and response shift are also shown in Table 2. Average recall bias ranged from -0.09 (patients with PTSD) to -0.004 (patients with TBI). Overall, recalled T1 EQ-5D-3 L was lower (-0.02) than the directly assessed EQ-5D-3 L, except for males and patients with a high educational level (all $p<0.05$ ). Pairwise comparisons showed that agreement between recall bias and response shift was good (ICC $=0.68, p<0.05)$. Multivariate linear regression analysis indicated that increasing PTSD symptoms were associated with recalling T1 EQ-5D-3 L as lower ('worse') than directly assessed EQ-5D-3 L at T1 (see Table 3). The EQ-5D-3 L dimensions that differed most frequently between the directly assessed EQ-5D-3 L at T1 and the recall test were usual activities $(36.5 \%$ of the respondents chose a different response option on the recall test), pain and/or other complaints (34.4\%), self-care (28.7\%) and anxiety/depression (27.5\%).

Mean response shift ranged from -0.12 (patients with an $I S S \geq 16$ ) to -0.04 (patients with a high educational level and patients older than 65 years). Multivariate linear regression analysis indicated that increasing 
Table 2 Mean EQ-5D-3 L summary score at T1, conventional change and retrospective change between EQ-5D-3 L summary scores at $\mathrm{T} 1$ and $\mathrm{T} 2$ and magnitude of recall bias and response shift

\begin{tabular}{|c|c|c|c|c|c|c|c|c|c|c|c|}
\hline & $\mathrm{n}$ & $\begin{array}{l}\text { Mean EQ- } \\
5 \mathrm{D} \text { T1 }\end{array}$ & $\begin{array}{l}\text { Mean EQ- } \\
5 \mathrm{D} \text { T2 }\end{array}$ & $\begin{array}{l}\text { Mean Then } \\
\text { Test }\end{array}$ & $\begin{array}{l}\text { Conventional } \\
\text { change }\end{array}$ & $\begin{array}{l}\text { Retrospective } \\
\text { change }\end{array}$ & $\mathrm{ICC}^{\mathrm{a}}$ & $\begin{array}{l}\text { Mean Recall } \\
\text { Test }\end{array}$ & $\begin{array}{l}\text { Recall } \\
\text { bias }\end{array}$ & $\begin{array}{l}\text { Response } \\
\text { shift }\end{array}$ & $\mathrm{ICC}^{\mathbf{b}}$ \\
\hline Total & $550^{c}$ & $\begin{array}{l}0.482 \\
(S D 0.30)\end{array}$ & $\begin{array}{l}0.735 \\
(\mathrm{SD} 0.24)\end{array}$ & $\begin{array}{l}0.419 \\
\text { (SD 0.35) }\end{array}$ & $\begin{array}{l}0.254 \\
(\mathrm{SD} 0.29)\end{array}$ & $\begin{array}{l}0.316 \\
\text { (SD 0.31) }\end{array}$ & $0.489^{*}$ & $\begin{array}{l}0.462 \\
\text { (SD 0.34) }\end{array}$ & $\begin{array}{l}-0.020 \\
(S D 0.30)\end{array}$ & $\begin{array}{l}-0.063 \\
(S D 0.31)\end{array}$ & $0.681^{*}$ \\
\hline \multicolumn{12}{|l|}{ Gender } \\
\hline Males & 308 & $\begin{array}{l}0.500 \\
(S D 0.31)\end{array}$ & $\begin{array}{l}0.741 \\
(S D 0.25)\end{array}$ & $\begin{array}{l}0.450 \\
\text { (SD 0.36) }\end{array}$ & $\begin{array}{l}0.242 \\
\text { (SD 0.31) }\end{array}$ & $\begin{array}{l}0.292 \\
\text { (SD 0.33) }\end{array}$ & $0.545^{*}$ & $\begin{array}{l}0.506 \\
\text { (SD 0.35) }\end{array}$ & $\begin{array}{l}0.007 \\
\text { (SD 0.31) }\end{array}$ & $\begin{array}{l}-0.050 \\
(S D 0.30)\end{array}$ & $0.705^{*}$ \\
\hline Females & 242 & $\begin{array}{l}0.459 \\
(\text { SD } 0.28)\end{array}$ & $\begin{array}{l}0.728 \\
\text { (SD 0.23) }\end{array}$ & $\begin{array}{l}0.380 \\
\text { (SD 0.33) }\end{array}$ & $\begin{array}{l}0.269 \\
(0.26)\end{array}$ & $\begin{array}{l}0.348 \\
\text { (SD 0.30) }\end{array}$ & $0.391^{*}$ & $\begin{array}{l}0.405 \\
\text { (SD 0.33) }\end{array}$ & $\begin{array}{l}-0.054 \\
\text { (SD 0.29) }\end{array}$ & $\begin{array}{l}-0.079 \\
(S D 0.31)\end{array}$ & $0.648^{*}$ \\
\hline \multicolumn{12}{|l|}{ Age } \\
\hline$<65$ years & 285 & $\begin{array}{l}0.452 \\
\text { (SD 0.29) }\end{array}$ & $\begin{array}{l}0.741 \\
\text { (SD 0.24) }\end{array}$ & $\begin{array}{l}0.373 \\
\text { (SD 0.34) }\end{array}$ & $\begin{array}{l}0.289 \\
\text { (SD 0.29) }\end{array}$ & $\begin{array}{l}0.369 \\
\text { (SD 0.32) }\end{array}$ & $0.516^{*}$ & $\begin{array}{l}0.425 \\
\text { (SD 0.34) }\end{array}$ & $\begin{array}{l}-0.028 \\
\text { (SD 0.31) }\end{array}$ & $\begin{array}{l}-0.080 \\
(S D 0.30)\end{array}$ & $0.749^{*}$ \\
\hline $65+$ years & 265 & $\begin{array}{l}0.513 \\
\text { (SD 0.30) }\end{array}$ & $\begin{array}{l}0.729 \\
\text { (SD 0.24) }\end{array}$ & $\begin{array}{l}0.469 \\
\text { (SD 0.35) }\end{array}$ & $\begin{array}{l}0.216 \\
\text { (SD 0.29) }\end{array}$ & $\begin{array}{l}0.260 \\
(S D 0.30)\end{array}$ & $0.435^{*}$ & $\begin{array}{l}0.501 \\
\text { (SD 0.35) }\end{array}$ & $\begin{array}{l}-0.012 \\
\text { (SD 0.29) }\end{array}$ & $\begin{array}{l}-0.044 \\
(S D 0.31)\end{array}$ & 0.608 \\
\hline \multicolumn{12}{|c|}{ Educational level $^{1}$} \\
\hline Low & 127 & $\begin{array}{l}0.503 \\
\text { (SD 0.33) }\end{array}$ & $\begin{array}{l}0.697 \\
\text { (SD 0.26) }\end{array}$ & $\begin{array}{l}0.443 \\
\text { (SD 0.37) }\end{array}$ & $\begin{array}{l}0.193 \\
\text { (SD 0.30) }\end{array}$ & $\begin{array}{l}0.254 \\
\text { (SD 0.30) }\end{array}$ & $0.439^{*}$ & $\begin{array}{l}0.489 \\
\text { (SD 0.35) }\end{array}$ & $\begin{array}{l}-0.015 \\
\text { (SD 0.32) }\end{array}$ & $\begin{array}{l}-0.060 \\
(S D 0.32)\end{array}$ & $0.661^{*}$ \\
\hline Middle & 214 & $\begin{array}{l}0.492 \\
(S D 0.30)\end{array}$ & $\begin{array}{l}0.740 \\
\text { (SD 0.25) }\end{array}$ & $\begin{array}{l}0.406 \\
\text { (SD 0.350) }\end{array}$ & $\begin{array}{l}0.248 \\
(S D 0.30)\end{array}$ & $\begin{array}{l}0.334 \\
\text { (SD 0.30) }\end{array}$ & $0.458^{*}$ & $\begin{array}{l}0.451 \\
\text { (SD 0.36) }\end{array}$ & $\begin{array}{l}-0.041 \\
(S D 0.32)\end{array}$ & $\begin{array}{l}-0.086 \\
(\text { SD } 0.31)\end{array}$ & $0.717^{*}$ \\
\hline High & 199 & $\begin{array}{l}0.447 \\
\text { (SD 0.27) }\end{array}$ & $\begin{array}{l}0.752 \\
\text { (SD 0.23) }\end{array}$ & $\begin{array}{l}0.403 \\
\text { (SD 0.34) }\end{array}$ & $\begin{array}{l}0.305 \\
\text { (SD 0.26) }\end{array}$ & $\begin{array}{l}0.349 \\
\text { (SD 0.33) }\end{array}$ & $0.543^{*}$ & $\begin{array}{l}0.448 \\
\text { (SD 0.33) }\end{array}$ & $\begin{array}{l}0.012 \\
\text { (SD 0.26) }\end{array}$ & $\begin{array}{l}-0.044 \\
\text { (SD 0.28) }\end{array}$ & $0.650^{*}$ \\
\hline \multicolumn{12}{|c|}{ Comorbidity status $^{2}$} \\
\hline $\begin{array}{l}\text { No } \\
\text { comorbidity }\end{array}$ & 231 & $\begin{array}{l}0.499 \\
\text { (SD 0.28) }\end{array}$ & $\begin{array}{l}0.784 \\
(S D 0.24)\end{array}$ & $\begin{array}{l}0.437 \\
\text { (SD 0.33) }\end{array}$ & $\begin{array}{l}0.285 \\
\text { (SD 0.28) }\end{array}$ & $\begin{array}{l}0.347 \\
\text { (SD 0.31) }\end{array}$ & $0.532^{*}$ & $\begin{array}{l}0.480 \\
\text { (SD 0.32) }\end{array}$ & $\begin{array}{l}-0.019 \\
(S D 0.30)\end{array}$ & $\begin{array}{l}-0.062 \\
(S D 0.29)\end{array}$ & $0.736^{*}$ \\
\hline Comorbidity & 309 & $\begin{array}{l}0.472 \\
\text { (SD 0.31) }\end{array}$ & $\begin{array}{l}0.702 \\
\text { (SD 0.24) }\end{array}$ & $\begin{array}{l}0.407 \\
\text { (SD 0.36) }\end{array}$ & $\begin{array}{l}0.230 \\
\text { (SD 0.29) }\end{array}$ & $\begin{array}{l}0.295 \\
\text { (SD 0.32) }\end{array}$ & $0.450^{*}$ & $\begin{array}{l}0.452 \\
\text { (SD 0.36) }\end{array}$ & $\begin{array}{l}-0.020 \\
(S D 0.30)\end{array}$ & $\begin{array}{l}-0.065 \\
\text { (SD 0.32) }\end{array}$ & $0.655^{*}$ \\
\hline \multicolumn{12}{|l|}{ ISS $^{3}$} \\
\hline ISS $<16$ & 513 & $\begin{array}{l}0.493 \\
\text { (SD 0.29) }\end{array}$ & $\begin{array}{l}0.741 \\
\text { (SD 0.24) }\end{array}$ & $\begin{array}{l}0.433 \\
\text { (SD 0.34) }\end{array}$ & $\begin{array}{l}0.248 \\
\text { (SD 0.29) }\end{array}$ & $\begin{array}{l}0.308 \\
\text { (SD 0.31) }\end{array}$ & $0.503^{*}$ & $\begin{array}{l}0.474 \\
\text { (SD 0.34) }\end{array}$ & $\begin{array}{l}-0.019 \\
\text { (SD 0.30) }\end{array}$ & $\begin{array}{l}-0.060 \\
\text { (SD 0.30) }\end{array}$ & $0.670^{*}$ \\
\hline ISS $>=16$ & 35 & $\begin{array}{l}0.350 \\
\text { (SD 0.30) }\end{array}$ & $\begin{array}{l}0.659 \\
\text { (SD 0.28) }\end{array}$ & $\begin{array}{l}0.229 \\
\text { (SD 0.40) }\end{array}$ & $\begin{array}{l}0.309 \\
\text { (SD 0.29) }\end{array}$ & $\begin{array}{l}0.429 \\
\text { (SD 0.36) }\end{array}$ & $0.315^{*}$ & $\begin{array}{l}0.289 \\
\text { (SD 0.38) }\end{array}$ & $\begin{array}{l}-0.061 \\
\text { (SD 0.34) }\end{array}$ & $\begin{array}{l}-0.120 \\
\text { (SD 0.39) }\end{array}$ & $0.740^{*}$ \\
\hline \multicolumn{12}{|l|}{ TBI } \\
\hline No TBI & 384 & $\begin{array}{l}0.432 \\
\text { (SD 0.28) }\end{array}$ & $\begin{array}{l}0.721 \\
\text { (SD 0.24) }\end{array}$ & $\begin{array}{l}0.383 \\
\text { (SD 0.33) }\end{array}$ & $\begin{array}{l}0.290 \\
\text { (SD 0.28) }\end{array}$ & $\begin{array}{l}0.338 \\
\text { (SD 0.31) }\end{array}$ & $0.461^{*}$ & $\begin{array}{l}0.428 \\
\text { (SD 0.33) }\end{array}$ & $\begin{array}{l}-0.004 \\
\text { (SD 0.30) }\end{array}$ & $\begin{array}{l}-0.048 \\
\text { (SD 0.31) }\end{array}$ & $0.656^{*}$ \\
\hline TBI & 166 & $\begin{array}{l}0.598 \\
\text { (SD 0.30) }\end{array}$ & $\begin{array}{l}0.768 \\
\text { (SD 0.25) }\end{array}$ & $\begin{array}{l}0.501 \\
\text { (SD 0.38) }\end{array}$ & $\begin{array}{l}0.171 \\
\text { (SD 0.28) }\end{array}$ & $\begin{array}{l}0.267 \\
\text { (SD 0.31) }\end{array}$ & $0.525^{*}$ & $\begin{array}{l}0.539 \\
\text { (SD 0.37) }\end{array}$ & $\begin{array}{l}-0.058 \\
\text { (SD 0.29) }\end{array}$ & $\begin{array}{l}-0.096 \\
\text { (SD 0.29) }\end{array}$ & $0.742^{*}$ \\
\hline \multicolumn{12}{|c|}{ PTSD symptoms ${ }^{4 d}$} \\
\hline No PTSD & 451 & $\begin{array}{l}0.490 \\
\text { (SD 0.29) }\end{array}$ & $\begin{array}{l}0.759 \\
\text { (SD 0.22) }\end{array}$ & $\begin{array}{l}0.423 \\
\text { (SD 0.34) }\end{array}$ & $\begin{array}{l}0.269 \\
\text { (SD 0.28) }\end{array}$ & $\begin{array}{l}0.337 \\
\text { (SD 0.31) }\end{array}$ & $0.518^{*}$ & $\begin{array}{l}0.471 \\
\text { (SD 0.34) }\end{array}$ & $\begin{array}{l}-0.019 \\
(S D 0.30)\end{array}$ & $\begin{array}{l}-0.068 \\
\text { (SD 0.29) }\end{array}$ & $0.684^{*}$ \\
\hline PTSD & 41 & $\begin{array}{l}0.345 \\
\text { (SD 0.35) }\end{array}$ & $\begin{array}{l}0.487 \\
\text { (SD 0.35) }\end{array}$ & $\begin{array}{l}0.270 \\
\text { (SD 0.43) }\end{array}$ & $\begin{array}{l}0.142 \\
\text { (SD 0.41) }\end{array}$ & $\begin{array}{l}0.216 \\
\text { (SD 0.35) }\end{array}$ & $0.294^{*}$ & $\begin{array}{l}0.252 \\
\text { (SD 0.36) }\end{array}$ & $\begin{array}{l}-0.093 \\
(S D 0.37)\end{array}$ & $\begin{array}{l}-0.075 \\
\text { (SD } 0.45)\end{array}$ & $0.780^{*}$ \\
\hline
\end{tabular}

SD standard deviation, ICC intraclass correlation coefficient, ISS injury severity score, TBI traumatic brain injury, PTSD posttraumatic stress disorder

${ }^{\text {a }}$ ICC corresponds to the correlation between conventional and retrospective change

${ }^{\mathrm{b}}$ ICC corresponds to the correlation between recall bias and response shift

'Patients who completed the EQ-5D at 1 week and 3 months and the then-test and recall-test for 1 week at 3 months after sustaining an injury

${ }^{d}$ PTSD symptoms measured with the Impact of Event Scale (IES) 3 months post-injury

${ }^{1} 10$ missing values, ${ }^{2} 10$ missing values, ${ }^{3} 2$ missing values, ${ }^{4} 58$ missing values

${ }^{*} p<0.05$

symptoms of PTSD were significantly associated with an increase in response shift (see Table 4). The EQ-5D-3 L dimensions that differed most frequently between the directly assessed EQ-5D-3 L at T1 and the then test that was used to assess response shift were pain and/or other complaints $35.6 \%$ of the respondents chose a different pain and/or other complaints response option on the then test), daily activities (34.5\%), self-care (31.3\%) and anxiety/depression (27.3\%). Response shift, with an average value of -0.06 , was significantly higher than recall bias $(Z=-4.5, p<0.05)$.

\section{EQ-VAS - conventional change versus retrospective change}

Table 5 shows the mean EQ-VAS score at T1 and the mean conventional change and retrospective change 
Table 3 Multivariate models for recall bias on the EQ-5D-3 $L$ summary score

\begin{tabular}{llll}
\hline & Initial model & & Final model \\
& Unstandardized B & $\boldsymbol{p}$-value & Unstandardized B \\
\hline Constant & 0.070 & 0.342 & 0.009 \\
Age $^{\text {a }}$ & 0.000 & 0.769 & \\
Sex (male/female) & -0.044 & 0.119 & \\
Education (dichotomized) & 0.025 & 0.379 & \\
Comorbidity (no/yes) & 0.031 & 0.312 & \\
TBI (no/yes) & -0.051 & 0.093 & \\
ISS $^{\text {a }}$ & 0.001 & 0.764 & \\
PTSD & -0.003 & 0.001 & -0.004 \\
F value & 2.96 & 0.05 & 13.73 \\
Adjusted R-square & 0.028 & & 0.026 \\
\hline
\end{tabular}

${ }^{a}$ Continuous variables

between EQ-VAS scores at T1 and T2. Mean EQ-VAS score improved from T1 (56.3; SD 20) to T2 (72.6; SD 17). A lower EQ-VAS score at T1 was associated with female gender, younger age, having an ISS $\geq 16$ and not having a TBI (all $p<0.05$ ). Individual agreement between retrospective and conventional change in EQVAS was fair (ICC $=0.483, p<0.05$ ) (see Table 5). Retrospective change in EQ-VAS score was significantly higher compared to conventional change $(Z=-2.1, p<$ $0.05)$. The difference between conventional and retrospective change in EQ-VAS was particularly large among patients with PTSD (difference $=-7.7, \mathrm{Z}=-2.4, p<$ 0.05 ), patients with an ISS $\geq 16$ (mean difference $=-6.6$, $\mathrm{Z}=-1.7, p=0.09$ ) and patients with a TBI (mean difference $=-4.7, Z=-2.9, p<0.05)$.

\section{EQ-VAS - recall bias versus response shift}

On average, the recalled T1 EQ-VAS was 0.6 lower ('worse') than directly assessed T1 EQ-VAS $(p<0.05)$.
The mean recall bias ranged from -7.3 for patients with PTSD to -0.3 for patients with comorbidity. Overall, recalled T1 EQ-5D-3 L was lower than the directly assessed EQ-5D-3 L, except for patients aged 65 and older, patients without TBI and patients with a high educational level (all $p<0.05$ ). Pairwise comparisons showed that agreement between recall bias and response shift was excellent $(\mathrm{ICC}=0.78, p<0.05)$. Multivariate linear regression analysis indicated that increasing PTSD symptoms and having TBI was significantly associated with a lower recalled T1 EQ-VAS compared to directly assessed EQVAS (see Table 6). With an average value of -1.6 , response shift was higher than recall bias, but this difference was not significant $(\mathrm{Z}=-0.635, p=0.53)$. Response shift was highest for patients with PTSD (mean: - 7.6), patients with an ISS $\geq 16$ (mean: -6.7) and patients with TBI (mean: -4.8 ) and lowest for patients without TBI (mean: -0.2 ) and patients older than 65 years (mean: - 0.3). Multivariate linear regression analysis indicated that

Table 4 Multivariate models for response shift based on the EQ-5D-3 L summary score

\begin{tabular}{llll}
\hline & Initial model & Final model \\
& Unstandardized B & $\boldsymbol{p}$-value & Unstandardized B \\
\hline Constant & -0.039 & 0.603 & -0.049 \\
Age $^{\text {a }}$ & $<0.001$ & 0.750 & \\
Sex (male/female) & -0.025 & 0.387 & \\
Education (dichotomized) & 0.037 & 0.201 & \\
Comorbidity (no/yes) & 0.002 & 0.945 & \\
TBI (no/yes) & -0.039 & 0.200 & \\
ISS $^{\text {a }}$ & 0.000 & 0.983 & \\
PTSD & -0.002 & 0.037 & -0.002 \\
F value & 1.40 & 0.20 & 5.83 \\
Adjusted R-square & 0.006 & & 0.010 \\
\hline
\end{tabular}

${ }^{\mathrm{a}}$ Continuous variables 
Table 5 Mean EQ-VAS score at T1, conventional change and retrospective change between EQ-VAS score at T1 and T2 and magnitude of recall bias and response shift

\begin{tabular}{|c|c|c|c|c|c|c|c|c|c|c|}
\hline & $\begin{array}{l}\text { EQ-VAS } \\
\text { T1 }\end{array}$ & $\begin{array}{l}\text { Mean EQ- } \\
5 \mathrm{D} \text { T2 }\end{array}$ & Mean Then Test & $\begin{array}{l}\text { Conventional } \\
\text { change }\end{array}$ & $\begin{array}{l}\text { Retrospective } \\
\text { change }\end{array}$ & $\mathrm{ICC}^{\alpha}$ & $\begin{array}{l}\text { Mean Recall } \\
\text { Test }\end{array}$ & $\begin{array}{l}\text { Recall } \\
\text { bias }\end{array}$ & $\begin{array}{l}\text { Response } \\
\text { shift }\end{array}$ & $\mathrm{ICC}^{\S}$ \\
\hline Total & $\begin{array}{l}56.3^{\$} \\
(S D 20)\end{array}$ & $\begin{array}{l}72.6 \\
\text { (SD 17.0) }\end{array}$ & $\begin{array}{l}54.7 \\
(S D 21.0)\end{array}$ & $\begin{array}{l}16.3 \\
(S D \text { 19) }\end{array}$ & $\begin{array}{l}17.9 \\
(S D 18)\end{array}$ & $0.483^{*}$ & $\begin{array}{l}55.7 \\
(S D \text { 21.0) }\end{array}$ & $\begin{array}{l}-0.62 \\
(S D 20)\end{array}$ & $\begin{array}{l}-1.59 \\
(S D \text { 19) }\end{array}$ & $0.783^{*}$ \\
\hline \multicolumn{11}{|l|}{ Gender } \\
\hline Males & $\begin{array}{l}58.3 \\
(S D 21)\end{array}$ & $\begin{array}{l}73.4 \\
\text { (SD 17.0) }\end{array}$ & $\begin{array}{l}56.4 \\
\text { (SD 22.5) }\end{array}$ & $\begin{array}{l}15.0 \\
(S D 12)\end{array}$ & $\begin{array}{l}17.0 \\
(S D 14)\end{array}$ & $0.464^{*}$ & $\begin{array}{l}57.6 \\
\text { (SD 22.5) }\end{array}$ & $\begin{array}{l}-0.74 \\
(S D 21)\end{array}$ & $\begin{array}{l}-1.94 \\
(S D 19)\end{array}$ & $0.793^{*}$ \\
\hline Females & $\begin{array}{l}53.8 \\
(S D \text { 19) }\end{array}$ & $\begin{array}{l}71.7 \\
\text { (SD 16.9) }\end{array}$ & $\begin{array}{l}52.7 \\
\text { (SD 18.8) }\end{array}$ & $\begin{array}{l}17.9 \\
(S D 19)\end{array}$ & $\begin{array}{l}19.0 \\
(S D 18)\end{array}$ & $0.503^{*}$ & $\begin{array}{l}53.3 \\
\text { (SD 18.7) }\end{array}$ & $\begin{array}{l}-0.46 \\
(S D \text { 17) }\end{array}$ & $\begin{array}{l}-1.14 \\
(S D \text { 19) }\end{array}$ & $0.768^{*}$ \\
\hline \multicolumn{11}{|l|}{ Age } \\
\hline$<65$ years & 54.1 (SD 20) & $\begin{array}{l}72.1 \\
\text { (SD 17.9) }\end{array}$ & $\begin{array}{l}51.4 \\
(S D 21.2)\end{array}$ & $\begin{array}{l}17.9 \\
(S D 19)\end{array}$ & $\begin{array}{l}20.7 \\
\text { (SD 19) }\end{array}$ & $0.459^{*}$ & $\begin{array}{l}51.7 \\
\text { (SD 20.9) }\end{array}$ & $\begin{array}{l}-2.46 \\
(S D 18)\end{array}$ & $\begin{array}{l}-2.74 \\
(S D 20)\end{array}$ & $0.740^{*}$ \\
\hline $65+$ years & $\begin{array}{l}58.7 \\
(S D 21)\end{array}$ & $\begin{array}{l}73.2 \\
\text { (SD 15.9) }\end{array}$ & $\begin{array}{l}58.3 \\
\text { (SD 20.2) }\end{array}$ & $\begin{array}{l}14.5 \\
(S D 20)\end{array}$ & $\begin{array}{l}14.8 \\
(S D \text { 16) }\end{array}$ & $0.498^{*}$ & $\begin{array}{l}60.0 \\
\text { (SD 20.2) }\end{array}$ & $\begin{array}{l}1.362 \\
(S D 21)\end{array}$ & $\begin{array}{l}-0.34 \\
(S D 18)\end{array}$ & $0.825^{*}$ \\
\hline \multicolumn{11}{|c|}{ Educational level $^{1}$} \\
\hline Low & 56.1 (SD 21) & $\begin{array}{l}69.7 \\
\text { (SD 16.9) }\end{array}$ & $\begin{array}{l}54.6 \\
\text { (SD 19.6) }\end{array}$ & $\begin{array}{l}13.6 \\
(S D 21)\end{array}$ & $\begin{array}{l}15.1 \\
(S D 19)\end{array}$ & $0.561^{*}$ & $\begin{array}{l}55.8 \\
\text { (SD 20.7) }\end{array}$ & $\begin{array}{l}-0.32 \\
(S D 20)\end{array}$ & $\begin{array}{l}-1.51 \\
(S D 19)\end{array}$ & $0.798^{*}$ \\
\hline Middle & 57.4 (SD 21) & $\begin{array}{l}74.3 \\
\text { (SD 17.2) }\end{array}$ & $\begin{array}{l}54.8 \\
\text { (SD 22.7) }\end{array}$ & $\begin{array}{l}17.0 \\
(S D 20)\end{array}$ & 19.6 (SD 18) & $0.402^{*}$ & $\begin{array}{l}55.7 \\
\text { (SD 22.5) }\end{array}$ & $\begin{array}{l}-1.71 \\
(S D 21)\end{array}$ & $\begin{array}{l}-2.61 \\
(S D 21)\end{array}$ & $0.773^{*}$ \\
\hline High & 55.1 (SD 20) & $\begin{array}{l}72.8 \\
\text { (SD 16.3) }\end{array}$ & $\begin{array}{l}54.3 \\
\text { (SD 20.0) }\end{array}$ & $\begin{array}{l}17.7 \\
(S D \text { 17) }\end{array}$ & $\begin{array}{l}18.5 \\
(S D 16)\end{array}$ & $0.519^{*}$ & $\begin{array}{l}55.4 \\
(S D \text { 19.6) }\end{array}$ & $\begin{array}{l}0.32 \\
(S D \text { 18) }\end{array}$ & $\begin{array}{l}-0.80 \\
(S D 16)\end{array}$ & $0.784^{*}$ \\
\hline \multicolumn{11}{|c|}{ Comorbidity status ${ }^{2}$} \\
\hline $\begin{array}{l}\text { No } \\
\text { comorbidity }\end{array}$ & $\begin{array}{l}57.3 \\
(S D 20)\end{array}$ & $\begin{array}{l}77.0 \\
\text { (SD 16.3) }\end{array}$ & $\begin{array}{l}56.2 \\
\text { (SD 20.9) }\end{array}$ & $\begin{array}{l}19.7 \\
(S D 20)\end{array}$ & $\begin{array}{l}20.8 \\
(S D \text { 19) }\end{array}$ & $0.524^{*}$ & $\begin{array}{l}56.7 \\
\text { (SD 20.5) }\end{array}$ & $\begin{array}{l}-0.58 \\
(S D 20)\end{array}$ & $\begin{array}{l}-1.06 \\
\text { (SD 19) }\end{array}$ & $0.772^{*}$ \\
\hline Comorbidity & $\begin{array}{l}55.6 \\
(S D 20)\end{array}$ & $\begin{array}{l}69.6 \\
\text { (SD 16.6) }\end{array}$ & $\begin{array}{l}53.9 \\
\text { (SD 20.8) }\end{array}$ & $\begin{array}{l}14.1 \\
(S D 19)\end{array}$ & $\begin{array}{l}15.7 \\
(S D 17)\end{array}$ & $0.421^{*}$ & $\begin{array}{l}55.3 \\
(S D 21.1)\end{array}$ & $\begin{array}{l}-0.27 \\
(S D 20)\end{array}$ & -1.676 (SD 19) & $0.793^{*}$ \\
\hline \multicolumn{11}{|l|}{$\mathrm{ISS}^{3}$} \\
\hline ISS $<16$ & $\begin{array}{l}57.3 \\
(S D 20)\end{array}$ & $\begin{array}{l}73.1 \\
\text { (SD 16.8) }\end{array}$ & $\begin{array}{l}55.9 \\
\text { (SD 20.3) }\end{array}$ & $\begin{array}{l}15.8 \\
(S D 19)\end{array}$ & $\begin{array}{l}17.11 \\
(S D \text { 17) }\end{array}$ & $0.503^{*}$ & $\begin{array}{l}56.6 \\
\text { (SD 20.4) }\end{array}$ & $\begin{array}{l}-0.69 \\
(\text { SD 19) }\end{array}$ & $\begin{array}{l}-1.31 \\
(S D 19)\end{array}$ & $0.802^{*}$ \\
\hline $\mathrm{ISS}>=16$ & $\begin{array}{l}44.6 \\
(S D 20)\end{array}$ & $\begin{array}{l}66.5 \\
\text { (SD 18.3) }\end{array}$ & $\begin{array}{l}37.9 \\
\text { (SD 23.6) }\end{array}$ & $\begin{array}{l}21.9 \\
(S D 23)\end{array}$ & $\begin{array}{l}28.5 \\
(S D 19)\end{array}$ & 0.199 & $\begin{array}{l}43.8 \\
\text { (SD 25.3) }\end{array}$ & $\begin{array}{l}-0.77 \\
(S D 29)\end{array}$ & $\begin{array}{l}-6.66 \\
(S D 27)\end{array}$ & $0.661^{*}$ \\
\hline \multicolumn{11}{|l|}{ TBI } \\
\hline No TBI & $\begin{array}{l}54.5 \\
(S D 20)\end{array}$ & $\begin{array}{l}71.8 \\
\text { (SD 17.0) }\end{array}$ & $\begin{array}{l}54.3 \\
\text { (SD 20.6) }\end{array}$ & $\begin{array}{l}17.3 \\
(S D 20)\end{array}$ & $\begin{array}{l}17.5 \\
(S D \text { 18) }\end{array}$ & $0.503^{*}$ & $\begin{array}{l}55.1 \\
\text { (SD 20.5) }\end{array}$ & $\begin{array}{l}0.57 \\
(S D 20)\end{array}$ & $\begin{array}{l}-0.22 \\
(S D 19)\end{array}$ & $0.787^{*}$ \\
\hline TBI & $\begin{array}{l}60.5 \\
(S D 21)\end{array}$ & $\begin{array}{l}74.4 \\
\text { (SD 16.7) }\end{array}$ & $\begin{array}{l}55.8 \\
\text { (SD 22.1) }\end{array}$ & $\begin{array}{l}13.9 \\
(S D 17)\end{array}$ & $\begin{array}{l}18.6 \\
(S D \text { 17) }\end{array}$ & $0.445^{*}$ & $\begin{array}{l}57.2 \\
(S D 22.2)\end{array}$ & $\begin{array}{l}-3.37 \\
(\text { SD 19) }\end{array}$ & $\begin{array}{l}-4.75 \\
\text { (SD 18) }\end{array}$ & $0.765^{*}$ \\
\hline \multicolumn{11}{|c|}{ PTSD symptoms ${ }^{4 \&}$} \\
\hline No PTSD & $\begin{array}{l}56.9 \\
(S D 20)\end{array}$ & $\begin{array}{l}73.7 \\
\text { (SD 16.3) }\end{array}$ & $\begin{array}{l}55.6 \\
(S D 21.1)\end{array}$ & $\begin{array}{l}16.8 \\
(S D 19)\end{array}$ & $\begin{array}{l}18.2 \\
(S D 18)\end{array}$ & $0.495^{*}$ & $\begin{array}{l}56.2 \\
\text { (SD 20.8) }\end{array}$ & $\begin{array}{l}-0.72 \\
(S D \text { 19) }\end{array}$ & $\begin{array}{l}-1.39 \\
(S D 18)\end{array}$ & $0.774^{*}$ \\
\hline PTSD & $\begin{array}{l}51.8 \\
(S D 21)\end{array}$ & $\begin{array}{l}58.1 \\
\text { (SD 17.7) }\end{array}$ & $\begin{array}{l}44.2 \\
\text { (SD 20.8) }\end{array}$ & $\begin{array}{l}6.2 \\
(S D 22)\end{array}$ & $\begin{array}{l}13.9 \\
(S D 19)\end{array}$ & $0.556^{*}$ & $\begin{array}{l}44.5 \\
\text { (SD 21.2) }\end{array}$ & $\begin{array}{l}-7.29 \\
(S D 20)\end{array}$ & $\begin{array}{l}-7.61 \\
(S D 19)\end{array}$ & $0.753^{*}$ \\
\hline
\end{tabular}

$S D$ standard deviation, ICC intraclass correlation coefficient, ISS injury severity score, TBI traumatic brain injury, PTSD posttraumatic stress disorder

a ICC corresponds to the correlation between conventional and retrospective change

§ ICC corresponds to the correlation between recall bias and response shift

${ }^{\$}$ Patients who completed the EQ-5D at 1 week and 3 months and the then-test and recall-test for 1 week at 3 months after sustaining an injury

\&PTSD symptoms measured with the Impact of Event Scale (IES) 3 months post-injury

${ }^{1} 10$ missing values, ${ }^{2} 10$ missing values, ${ }^{3} 2$ missing values, ${ }^{4} 58$ missing values

increasing PTSD symptoms and having TBI was associated with response shift (see Table 7).

\section{Discussion}

Our study showed that retrospective change in HRQL exceeded conventional change and that, at the individual level, agreement between conventional and retrospective change was only fair for both the EQ-5D-3 L summary score and the EQ-VAS. Response shift, more than recall bias, modified the reported retrospective outcome.

The relative magnitude of recall bias and response shift was higher when measured with the EQ-VAS 
Table 6 Multivariate models for response shift based on the EQ-VAS

\begin{tabular}{llll}
\hline & Initial model & & Final model \\
& Unstandardized B & $\boldsymbol{p}$-value & Unstandardized B \\
\hline Constant & -5.977 & 0.342 & 0.482 \\
Age $^{\text {a }}$ & 0.059 & 0.769 & \\
Sex (male/female) & 2.146 & 0.119 & \\
Education (dichotomized) & 1.482 & 0.674 \\
Comorbidity (no/yes) & 0.351 & 0.312 & \\
TBI (no/yes) & -3.922 & 0.093 & \\
ISS $^{\text {a }}$ & -0.138 & 0.764 & -4.037 \\
PTSD & -0.142 & 0.001 & \\
F value & 2.15 & 0.038 & -0.135 \\
Adjusted R-square & 0.017 & & 0.09 \\
\hline
\end{tabular}

${ }^{a}$ Continuous variables

compared to the EQ-5D-3 L summary score. This may be due to the fact that the restricted range of responses of the EQ-5D-3 L may lead to smaller variability in scores compared to the continuous EQ-VAS [13]. The subjectivity of the scale, which is higher for VAS compared to the classification-like EQ-5D-3 L, played a much smaller role than expected. We expected that the individual agreement between conventional and retrospective change would be higher for the EQ-5D-3 L summary score compared to the EQ-VAS; however, our findings showed that the individual agreement was similar.

In agreement to our expectations, response shift was higher among trauma patients with severe injuries (ISS $\geq$ 16). This indicates that high impact trauma requires more adaptation to one's health status compared to less severe trauma. Our findings also showed that, relatively shortly after sustaining injury, the magnitude of response shift is already quite large. This is in agreement with a study that assessed response shift among individuals with stroke and that found evidence of similarly large magnitude of response shift 24 weeks post-stroke [35].

Conversely to our expectations we did not find that the size of recall bias was higher in older respondents compared to their younger counterparts. This finding may be explained by participation bias. In our study, response rate was rather low and, possibly, the elderly that did participate may not have been representative of elder trauma patients in the sense that the elderly respondents may experience less memory problems compared to the elderly non-respondents.

Table 7 Multivariate models for recall bias based on the EQ-VAS

\begin{tabular}{llll}
\hline & Initial model & & Final model \\
& Unstandardized B & $\boldsymbol{p}$-value & Unstandardized B \\
\hline Constant & -8.476 & 0.069 & 1.127 \\
Age $^{\text {a }}$ & 0.083 & 0.178 & \\
Sex & 1.727 & 0.331 & \\
Education (dichotomized) & 1.889 & 0.299 & \\
Comorbidity (yes/no) & 1.400 & 0.474 & \\
TBI (yes/no) & -3.685 & 0.055 & -3.808 \\
ISS $^{\text {a }}$ & 0.100 & 0.521 & \\
PTSD $^{\text {a }}$ & -0.147 & 0.022 & -0.134 \\
F value & 2.16 & 0.036 & 4.62 \\
Adjusted R-square & 0.017 & & 0.015 \\
\hline
\end{tabular}

${ }^{\mathrm{a}}$ Continuous variables 
We did find that response shift increased with increasing PTSD symptoms. This may indicate that symptoms of PTSD affect cognitive dissonance between the actual health state of the respondent and the desired health state. Second, both TBI and PTSD were positively associated with recall bias. Similarly to TBI, PTSD is associated with impairments in cognitive functioning [36-38]. Our findings clearly confirm that cognitive impairment is a non-trivial factor in research where recall bias may occur.

Agreement between conventional and retrospective change in HRQL was similar to the agreement reported by McPhail et al. and, similarly to McPhail et al., we found a higher retrospective change than conventional change for both the EQ-5D-3 L summary score and EQ5D-3 L [10]. However, McPhail et al. reported a much larger difference between conventional and retrospective change and higher recall bias. This difference in findings may be explained by the difference in study population and/or timing of HRQL assessments. McPhail et al. studied hospitalized elderly, whereas we studied hospitalized trauma patients aged 18 and older. Higher age of the respondents may have contributed to the difference in conventional and retrospective change and magnitude of recall bias, although our study did not show important differences between conventional and retrospective change, recall bias or response shift in younger versus older trauma patients. With regards to the timing of HRQL assessment, McPhail et al. measured HRQL immediately after hospital admission (T1) and immediately after discharge (T2), whereas our first measurement of HRQL was 1 week post-injury. As a result, the change in HRQL at T1 and T2, as measured by McPhail et al., may have been much larger and subsequently also the contribution of recall bias and response shift to the difference between conventional and retrospective change in HRQL.

\section{Strengths and limitations}

Strengths of our study were the meticulous protocol and the regional coverage, with a high number of respondents. The combined use of subjective and a classification-like scales reinforced analytical opportunities as shown. The high number of respondents allowed us to test for differences between conventional and retrospective change in HRQL and assess recall bias and response shift for specific subgroups of trauma patients, such as trauma patients with severe injury and patients with TBI. The use of the EQ-5D-3 L and EQ-VAS allowed us to compare differences between conventional and retrospective change in HRQL and contribution of recall bias and response shift on a subjective scale and a classification like scale.
A limitation of our study was the uniform follow-up time (3 months), which limited conclusions on durationdependence of the bias effects. In our study, the time between first and second measurement was 3 months. Stronger recall bias may be expected over longer periods, with perhaps recall becoming more important than response shift [11]. If e.g. 12 months instead of 3 months has been chosen as interval, the effect of recall bias might have been more pronounced.

A second limitation of our study was that the T2 survey included the EQ-5D-3 L and EQ-VAS for the direct measurement of current HRQL as well as the recall and then-test regarding the respondents' HRQL at T1. This was a challenging task. This meant that the respondents had to fill out several similarly formulated questions. This may have affected both the number of respondents with complete responses, as well as the quality of the responses. Since we administered stand-alone paper-and-pencil surveys, we were not able to verify if the respondents understood the recall and then test and the difference between these two questionnaires.

A third limitation is the use of EQ-5D-3 L rather than the EQ-5D-5 L. As the EQ-5D-5 L has five response options instead of three with more sensitivity and precision, contrasts could have been larger in that case [39-41]. For future studies that aim to investigate recall bias and response shift we recommend to use the EQ-5D-5 L.

\section{Implications for clinical practice}

The findings of our study confirm that, in our sample of trauma patients, there was disagreement between conventional and retrospective change of HRQL and that recall bias and response shift both contributed to this difference. This is important to take into account when change in HRQL is used to evaluate health interventions in this patient group. Whether conventional or retrospective change should be used for the evaluation depends strongly on the aims of the intervention and the characteristics of the patients, as well as which perspective of change is the most important to various stakeholders, as was also pointed out by McPhail et al. [10].

\section{Conclusions}

We conclude that, compared to recall bias, response shift contributed more to the disagreement between conventional and retrospective change in EQ-5D-3 L summary score and EQ-VAS. Predictable subgroups of trauma patients were more susceptible to recall bias and response shift, such as patients who sustained TBI and patients with PTSD symptoms 3 months post-injury. 


\section{Abbreviations}

AIS: Abbreviated Injury Scale; ANOVA: One-way analysis of variance; BIOS: Brabant Injury Outcome Surveillance; ED: Emergency Department; HRQL: Health-related quality of life; ICC: Intraclass correlation coefficient; ICU: Intensive Care unit; IES: Impact of event scale; ISS: Injury Severity Score; IQR: Interquartile range; PTSD: Posttraumatic stress disorder; SD: Standard deviation; T1: Postal questionnaire 1 week after the initial treatment of the injury; T2: Postal questionnaire 3 months after the initial treatment of the injury; TBI: Traumatic brain injury; VAS: Visual analogue scale

\section{Authors' contributions}

$J H, G B, M d J$ and SP conceptualized the design of the study. MdJ and SP coordinated the collection of the data. IS and JH analysed the data. JH, IS, GB, MdJ and SP interpreted the data. JH, IS and GB drafted the manuscript. $J H, G B, I S, M d J$ and SP revised the manuscript critically for important intellectual content. IS, GB, MdJ and SP have given final approval of the version of the manuscript that was submitted to be published. Each author agreed to be accountable for all aspects of the work in ensuring that questions related to the accuracy or integrity of any part of the work are appropriately investigated and resolved. The author(s) read and approved the final manuscript.

\section{Funding}

This study was funded by the EuroQoL group (Grant number EQ Project 2016160).

\section{Availability of data and materials}

The datasets used and/or analysed during the current study are available from the corresponding author on reasonable request.

\section{Ethics approval and consent to participate}

This study was approved by the Medical Ethics Committee Brabant, the Netherlands. Patients needed to give permission for participation in the BIOS study. Informed consent forms were sent together with the T1 questionnaire or together with questionnaires sent at later time points when the patient did not respond to the $\mathrm{T} 1$ questionnaire.

\section{Consent for publication}

Not applicable.

\section{Competing interests}

This study was funded by the EuroQoL group (Grant number EQ Project 2016160), of which GJB is a member.

\section{Author details}

${ }^{1}$ Department of Public Health, Erasmus MC University Medical Center Rotterdam, P.O. Box 2040, 3000, CA, Rotterdam, The Netherlands. ${ }^{2}$ Association of Dutch Burn Centres, Maasstad Hospital, Rotterdam, The Netherlands. ${ }^{3}$ Department Trauma TopCare, ETZ Hospital, Tilburg, The Netherlands.

\section{Received: 10 April 2019 Accepted: 11 May 2020}

\section{Published online: 27 May 2020}

\section{References}

1. Polinder S, Haagsma JA, Belt E, Lyons RA, Erasmus V, Lund J, van Beeck EF. A systematic review of studies measuring health-related quality of life of general injury populations. BMC Public Health. 2010;10:783.

2. Sullivan $\mathrm{M}$. The new subjective medicine: taking the patient's point of view on health care and health. Soc Sci Med. 2003;56:1595-604.

3. Fanning J, Walkup MP, Ambrosius WT, Brawley LR, Ip EH, Marsh AP, Rejeski WJ. Change in health-related quality of life and social cognitive outcomes in obese, older adults in a randomized controlled weight loss trial: does physical activity behavior matter? J Behav Med. 2018;41:299-308.

4. Liimatta $\mathrm{H}$, Lampela P, Laitinen-Parkkonen P, Pitkala KH. Effects of preventive home visits on health-related quality-of-life and mortality in home-dwelling older adults. Scand J Prim Health Care. 2019;37:90-7.

5. Dhanda R, Varghese D, Nadipelli VR, Fava M, Joshi N, Solem CT, Graham JA, Learned SM, Heidbreder C. Patient-reported outcomes in schizophrenia patients treated with once-monthly extended-release risperidone in a longterm clinical study. Patient Prefer Adherence. 2019;13:1037-50.
6. Khalil JG, Smuck M, Koreckij T, Keel J, Beall D, Goodman B, Kalapos P, Nguyen D, Garfin S, Investigators IT. A prospective, randomized, multicenter study of intraosseous basivertebral nerve ablation for the treatment of chronic low back pain. Spine J. 2019;19:1620-32.

7. Brahmer JR, Rodriguez-Abreu D, Robinson AG, Hui R, Csoszi T, Fulop A, Gottfried M, Peled N, Tafreshi A, Cuffe S, et al. Health-related quality-of-life results for pembrolizumab versus chemotherapy in advanced, PD-L1positive NSCLC (KEYNOTE-024): a multicentre, international, randomised, open-label phase 3 trial. Lancet Oncol. 2017;18:1600-9.

8. Gabbe BJ, Simpson PM, Harrison JE, Lyons RA, Ameratunga S, Ponsford J, Fitzgerald M, Judson R, Collie A, Cameron PA. Return to work and functional outcomes after major trauma. Ann Surg. 2016;263:623-32.

9. Crosby RD, Kolotkin RL, Williams GR. Defining clinically meaningful change in health-related quality of life. J Clin Epidemiol. 2003;56:395-407.

10. McPhail S, Haines T. Response shift, recall bias and their effect on measuring change in health-related quality of life amongst older hospital patients. Health Qual Life Outcomes. 2010;8:65.

11. Schmier JK, Halpern MT. Patient recall and recall bias of health state and health status. Expert Rev Pharmacoecon Outcomes Res. 2004;4:159-63.

12. Stull DE, Leidy NK, Parasuraman B, Chassany O. Optimal recall periods for patient-reported outcomes: challenges and potential solutions. Curr Med Res Opin. 2009;25:929-42.

13. Kwong E, Neuburger J, Black N. Agreement between retrospectively and contemporaneously collected patient-reported outcome measures (PROMs) in hip and knee replacement patients. Qual Life Res. 2018;27(7):1845-54.

14. Howell J, Xu M, Duncan CP, Masri BA, Garbuz DS. A comparison between patient recall and concurrent measurement of preoperative quality of life outcome in total hip arthroplasty. J Arthroplast. 2008;23:843-9.

15. Topp J, Andrees V, Heesen C, Augustin M, Blome C. Recall of health-related quality of life: how does memory affect the SF-6D in patients with psoriasis or multiple sclerosis? A prospective observational study in Germany. BMJ Open. 2019;9:e032859.

16. Litwin MS, McGuigan KA. Accuracy of recall in health-related quality-of-life assessment among men treated for prostate cancer. J Clin Oncol. 1999;17: 2882-8.

17. Haagsma J, Bonsel $\mathrm{G}$, de Jongh $\mathrm{M}$, Polinder $\mathrm{S}$. Agreement between retrospectively assessed health-related quality of life collected 1 week and 12 months post-injury: an observational follow-up study. Health Qual Life Outcomes. 2019;17:70.

18. Silverberg ND, Iverson GL, Brubacher JR, Holland E, Hoshino LC, Aquino A, Lange RT. The nature and clinical significance of Preinjury recall Bias following mild traumatic brain injury. J Head Trauma Rehabil. 2016;31:38896.

19. Schwartz CE, Sprangers MAG. Methodological approaches for assessing response shift in longitudinal health-related quality-of-life research. Soc Sci Med. 1999;48:1531-48.

20. Sprangers MAG, Schwartz CE. Integrating response shift into health-related quality of life research: a theoretical model. Soc Sci Med. 1999:48:1507-15.

21. Schwartz CE, Sprangers MAG, Carey A, Reed G. Exploring response shift in longitudinal data. Psychol Health. 2003;19:51-69.

22. de Jongh MAC, Kruithof N, Gosens T, van de Ree CLP, de Munter L, Brouwers L, Polinder S, Lansink KWW. Prevalence, recovery patterns and predictors of quality of life and costs after non-fatal injury: the Brabant injury outcome surveillance (BIOS) study. Inj Prev. 2017;23:59.

23. Centraal Bureau voor de Statistiek. Permanent onderzoek leefsituatie. Voorburg/Heerlen: Statistics Netherlands (CBS); 2003.

24. Mosby. Mosby's Medical Dictionary. 9th ed; 2012.

25. Brooks R, Group E. EuroQol: the current state of play. Health Policy. 1996;37: 53-72.

26. Rabin R, Fd C. EQ-SD: a measure of health status from the EuroQol group. Ann Med. 2001;33:337-43.

27. Dolan P. Modeling valuations for EuroQol health states. Med Care. 1997: 1095-108.

28. Horowitz M, Wilner N, Alvarez W. Impact of event scale: a measure of subjective stress. Psychosom Med. 1979;41:209-18.

29. Wohlfarth TD, van den Brink W, Winkel FW, ter Smitten M. Screening for posttraumatic stress disorder: an evaluation of two self-report scales among crime victims. Psychol Assess. 2003;15:101-9.

30. Baker SP, o'Neill B, Haddon W Jr, Long WB. The injury severity score: a method for describing patients with multiple injuries and evaluating emergency care. J Trauma. 1974;14:187-96. 
31. Gennarelli TA, Wodzin E. AIS 2005: A contemporary injury scale. Injury. 2006(37):1083-91.

32. Boyd CR, Tolson MA, Copes WS. Evaluating trauma care: the TRISS method. Trauma score and the injury severity score. J Trauma. 1987;27:370-8

33. Bartko JJ. The intraclass correlation coefficient as a measure of reliability. Psychol Rep. 1966;19:3-11.

34. Cicchetti DV. Guidelines, criteria, and rules of thumb for evaluating normed and standardized assessment instruments in psychology. Psychol Assess. 1994;6:284.

35. Ahmed S, Mayo NE, Wood-Dauphinee S, Hanley JA, Cohen SR. Response shift influenced estimates of change in health-related quality of life poststroke. J Clin Epidemiol. 2004;57:561-70.

36. Aupperle RL, Melrose AJ, Stein MB, Paulus MP. Executive function and PTSD: disengaging from trauma. Neuropharmacology. 2012;62:686-94.

37. Qureshi SU, Long ME, Bradshaw MR, Pyne JM, Magruder KM, Kimbrell T, Hudson TJ, Jawaid A, Schulz PE, Kunik ME. Does PTSD impair cognition beyond the effect of trauma? J Neuropsychiatry Clin Neurosci. 2011;23:16-28.

38. Schuitevoerder S, Rosen JW, Twamley EW, Ayers CR, Sones H, Lohr JB, Goetter EM, Fonzo GA, Holloway KJ, Thorp SR. A meta-analysis of cognitive functioning in older adults with PTSD. J Anxiety Disord. 2013;27:550-8.

39. Agborsangaya CB, Lahtinen M, Cooke T, Johnson JA. Comparing the EQ-5D $3 \mathrm{~L}$ and $5 \mathrm{~L}$ : measurement properties and association with chronic conditions and multimorbidity in the general population. Health Qual Life Outcomes. 2014;12:74.

40. Janssen MF, Bonsel GJ, Luo N. Is EQ-5D-5L better than EQ-5D-3L? A headto-head comparison of descriptive systems and value sets from seven countries. Pharmacoeconomics. 2018;36:675-97.

41. Janssen MF, Pickard AS, Golicki D, Gudex C, Niewada M, Scalone L, Swinburn P, Busschbach J. Measurement properties of the EQ-5D-5L compared to the EQ-5D-3L across eight patient groups: a multi-country study. Qual Life Res. 2013;22:1717-27.

\section{Publisher's Note}

Springer Nature remains neutral with regard to jurisdictional claims in published maps and institutional affiliations.

Ready to submit your research? Choose BMC and benefit from:

- fast, convenient online submission

- thorough peer review by experienced researchers in your field

- rapid publication on acceptance

- support for research data, including large and complex data types

- gold Open Access which fosters wider collaboration and increased citations

- maximum visibility for your research: over $100 \mathrm{M}$ website views per year

At $\mathrm{BMC}$, research is always in progress.

Learn more biomedcentral.com/submissions 